\title{
Supervivencia de los pacientes adultos con leucemia mieloide aguda en el Departamento de Hematología del Hospital Pablo Tobón Uribe entre los años 2004 y 2010
}

- Laura María Díaz Correa, Camilo Alberto Madrid Muñoz, Juan Felipe Combariza Vallejo, Kenny Mauricio Gálvez Cárdenas, Viviana Olaya Colorado, Jorge Hernando Donado Gómez

\section{Resumen}

\begin{abstract}
Antecedentes. La leucemia mieloide aguda (LMA) es una neoplasia rara, con una edad media de presentación de 60 años, con marcada heterogeneidad, tanto en respuesta al tratamiento como en supervivencia. Los avances en la comprensión de su fisiopatología no han dado lugar a mejoras en la supervivencia libre de enfermedad y supervivencia global de adultos con esta enfermedad.
\end{abstract}

Objetivo. Describir supervivencia de pacientes con LMA manejados en el Hospital Pablo Tobón Uribe (HPTU) durante el período entre enero de 2004 y junio de 2010.

Metodología. Estudio observacional descriptivo de serie de casos, en pacientes con LMA diagnosticados y tratados en el HPTU entre los años 2004 y 2010.
Resultados. Cincuenta y tres casos de LMA, el 62,2\% eran mujeres. La mediana de edad fue de 55 años. La mediana de leucocitos al diagnóstico fue de 9.300 . Cinco casos $(9,43 \%)$ fueron secundarios a síndromes mielodisplásicos. Cincuenta y un pacientes $(96,22 \%)$ recibieron tratamiento quimioterápico. El esquema de tratamiento más frecuente fue idarrubicina más citarabina $7 \times 3$ en 39 pacientes $(90,69 \%)$. Veintiocho pacientes $(52,8 \%)$ alcanzaron respuesta completa a la quimioterapia de inducción; 3 pacientes fueron llevados a trasplante alogénico; 5 pacientes murieron durante la inducción, 3 de ellos mayores de 60 años. La mediana de supervivencia global fue de 348 días. La mediana de supervivencia libre de enfermedad fue de 404 días. La tasa de supervivencia global a un año fue del $49 \%$.

Conclusión. Serie de 53 pacientes adultos con LMA. Encontramos tiempos de supervivencia global similares a los de reportes mundiales.

\section{Caracterización morfológica e inmunofenotípica de la leucemia mieloide crónica}

\author{
Iván Bravo, Jaisury Arango, Natalia Olaya, Leonardo Fierro, Roberto Jaramillo
}

Universidad del Valle, Unidad de Diagnóstico Hematooncológico.

\begin{abstract}
La leucemia mieloide crónica (LMC) hace parte de las neoplasias mieloproliferativas crónicas, que para su diagnóstico requieren un equipo multidisciplinario compuesto por hematooncólogo, patólogo, laboratoristas clínicos, e involucra múltiples metodologías de laboratorio para definir un diagnóstico y pronóstico específico.

La citometría de flujo es una herramienta útil para la evaluación inmunofenotípica multiparamétrica, que evalúa la diferenciación y maduración de cada línea hematopoyética, y en el caso de la LMC, permite evaluar la maduración de la línea mieloide con el análisis de la expresión de distintos clústeres de diferenciación involucrados, así como la expresión aberrante de diversos marcadores de línea no linfoide.
\end{abstract}

Se presentan 17 casos con diagnóstico clínico y molecular de LMC entre junio de 2012 a enero de 2013 remitidos a la Unidad de Diagnóstico Hematooncológico en Cali. Se estudiaron las alteraciones de la expresión de los distintos anticuerpos de diferenciación mieloide en la población de neutrófilos teniendo en cuenta los distintos estadios de maduración.

En todos los casos se encontró neutrofilia con incremento de eosinófilos y basófilos, y alteraciones en la maduración de línea neutrófilo con expresión alterada para los clústeres de diferenciación CD13, CD11b, CD16 y CD10, principalmente, con expresión aberrante de CD56.

La citometría de flujo es una metodología diagnóstica que permite un análisis rápido y confiable de casos sospechosos de LMC, demostrando bloqueos de la maduración de los neutrófilos, evaluando la expresión de marcadores de diferenciación en los distintos estadios, así como también define la fase exacta de la enfermedad según el conteo de blastos. 